\title{
Transmissão de Sauroplasma sp. (Piroplasmorida: Haemohormidiidae) pela sanguessuga Unoculubranchiobdella expansa (Hirudinea: Ozobranchidae) em Podocnemis expansa (Tartaruga da Amazônia)
}

A espécie Podocnemis expansa possui grande importância para as comunidades tradicionais e indígenas da região amazônica. Possui o título de maior quelônio de água doce da América do Sul podendo chegar a pesar até $60 \mathrm{~kg}$, animal predominantemente herbívoro podendo aceitar carne e peixe em sua dieta quando em cativeiro. Assim como outros organismos, são suscetíveis a diversos parasitas, sendo os endoparasitos mais frequentes os nematóides, trematóides, monógenos, cestóides e protozoários e dentre os ectoparasitas, os hirudíneos. Esta pesquisa tem como objetivo verificar a transmissão experimental dos hemoparasitos Haemogregarina sp. e Sauroplasma sp. para Podocnemis expansa mediante repasto da sanguessuga Unoculubranchiobdella expansa. Os indivíduos capturados apresentaram prevalência de $100 \%$ quanto ao parasitismo por sanguessugas e $64,7 \%$ da população estudada estava parasitada por Sauroplasma sp. A intensidade de sanguessuga foi significativamente correlacionada com o índice de condição corporal ( $n=17 ; r s=0.812 ; p \leq 0.001$ ). Não houve correlação significativa entre a prevalência e a intensidade sanguessuga $(\chi 2=2.1604 ; d f=1 ; p=0.1416)$. Os filhotes submetidos à infecção, todos apresentaram Sauroplasma, não houve infecção de Hemogregarina.

Transmission of Sauroplasma sp. (Piroplasmorida: Haemohormidiidae) by the leech Unoculubranchiobdella expansa (Hirudinea: Ozobranchidae) in Podocnemis expansa (Amazonian

\section{Turtle)}

The species Podocnemis expansa has great importance for the traditional and indigenous communities of the Amazon region. It possesses the title of greater freshwater chelonium of South America being able to weigh up to $60 \mathrm{~kg}$, predominantly herbivorous animal being able to accept meat and fish in its diet when in captivity. Like other organisms, they are susceptible to several parasites, with the most frequent endoparasites being nematodes, trematóides, monogens, cestodes and protozoa and among the ectoparasites, the hirudíneos. This research aims to verify the experimental transmission of Haemogregarina sp. Hemoparasites. And Sauroplasma sp. For Podocnemis expansa by repasto of leech Unoculubranchiobdella expansa. The individuals captured had a $100 \%$ prevalence regarding leech parasitism and $64.7 \%$ of the studied population was parasitized by Sauroplasma $\mathrm{sp}$. Leech intensity was significantly correlated with body condition index ( $\mathrm{n}=17$ $r s=0.812 ; p \leq 0.001)$. There was no significant correlation between prevalence and leech intensity $(\chi 2=2.1604 ; d f=1 ; p=0.1416)$. The pups submitted to the infection, all presented Sauroplasma, there was no Hemogregarin infection.

Keywords: Sauroplasma; Podocnemis expansa; Leech.

Topic: Fundamentos de Biodiversidade

Reviewed anonymously in the process of blind peer.
Received: $14 / 12 / 2017$

Approved: 24/01/2018
Aluísio Vasconcelos Carvalho (iD)

Faculdade Guaraí, Brasil

http://lattes.cnpq.br/5200758055263996

http://orcid.org/0000-0002-3793-3133

aluisiovasconcelos@gmail.com

Adriana Malvasio

Universidade Federal do Tocantins, Brasil

http://lattes.cnpq.br/9694032726460437

malvasio@uft.edu.br

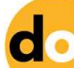

DOI: 10.6008/CBPC2179-6858.2018.002.0005
Referencing this:

CARVALHO, A. V.; MALVASIO, A.. Transmissão de Sauroplasma sp. (Piroplasmorida: Haemohormidiidae) pela sanguessuga Unoculubranchiobdella expansa (Hirudinea: Ozobranchidae) em Podocnemis expansa (Tartaruga da Amazônia). Revista Ibero Americana de Ciências Ambientais, v.9, n.2, p.41-53, 2018. DOI: http://doi.org/10.6008/CBPC2179-6858.2018.002.0005 


\section{INTRODUÇÃO}

O parasitismo é uma relação direta e estreita entre dois organismos bem determinados: o hospedeiro e o parasita, vivendo o segundo à custa do primeiro. Organismos parasitas devem manter seus respectivos hospedeiros vivos, pois necessitam de condições ótimas para sua sobrevivência, uma vez que esses últimos fornecem abrigo, nutrição e condições para a reprodução dos parasitos (PESSÔA et al., 1982; POUGH et al., 2004; LEGLER et al., 2013). Nos quelônios, geralmente, é possível encontrar endoparasitos na cavidade oral, bexiga urinária, pulmões, intestino, cavidade nasal, faringe, estômago e esôfago (MURPHY et al., 1983). Sendo os endoparasitos mais frequentes os nematóides, trematóides, monógenos, cestóides e protozoários (POUGH et al., 2004).

Quanto a ectoparasitos, é possível encontrar sanguessugas (Hirudinea) em animais juvenis e adultos, vetores importantes na transmissão de protozoários patógenos tais como Haemogregarina sp (ROSSOW et al., 2013). A família Haemogregarinidae contém seis gêneros: Haemogregarina, Karyolysus, Hepatozoon, Desseria, Hemolivia e Cyrilia (DAVIES et al., 2000). As hemogregarinas (Haemogregarina) seguem o ciclo de vida padrão do filo Apicomplexa, com reprodução sexuada no vetor, que no caso de $P$. expansa provavelmente é o grupo dos hirudíneos (sanguessuga), e reprodução assexuada no hospedeiro vertebrado, o quelônio. Algumas hemogregarinas têm um largo alcance em hospedeiros, tanto invertebrados do grupo dos artrópodes, tais como mosquitos (Hexapoda), ácaros e carrapatos (Arachnida), como vertebrados do grupo das répteis serpentes, lagartos (Squamata) e jacarés (Crocodilianos) (LEVINE, 1982; BALL, 1973).

Os parasitos do gênero Sauroplasma (TOIT, 1937) pertencem a ordem Piroplasmida, e fazem parte da família Haemohormidiidae, juntamente com os gêneros Serpentoplasma e Haemohormidium (LEVINE, 1980; READEL et al., 2008). De acordo com Toit (1937), o protozoário possui forma arredondada ou irregular, onde a forma típica é a de um anel com um grande vacúolo central associado a grânulos de cromatina. A reprodução ocorre por fissão binária ou brotamento. Piroplasmas podem aparecer em eritrócitos como pequenas inclusões medindo entre 1,1-5,0 × 1.0- $4.3 \mu \mathrm{m}$ (LAINSON et al., 1971; BENNET et al., 1976).

A ocorrência de Sauroplasma em répteis é bastante comum e com ampla distribuição geográfica, onde formas parasitárias têm sido registradas em diferentes famílias de lagartos na Europa, África, Ásia e nas Américas (TELFORD, 2016; UILENBERG et al., 1966; FRANK, 1974; ALBERTS et al., 1998). Entretanto, seu ciclo de vida, patogenicidade e vetores são desconhecidos (DAVIES et al., 2000; TELFORD, 2016). É provável que os vetores para répteis terrestres sejam carrapatos e ácaros devido à presença destes em lagartos infectados mas para répteis aquáticos o vetor é totalmente desconhecido (PIENAAR, 1962; UILENBERG et al., 1966; ALBERTS et al., 1998; READEL et al., 2008; TELFORD, 1984).

Contudo, existe a possibilidade de que as sanguessugas estejam envolvidas na transmissão de Sauroplasma em quelônios aquáticos (ARMOND, 2008; PICELLI et al., 2015). Tais ectoparasitos são relativamente comuns em répteis aquáticos (SAWYER, 1986; LIGHT et al., 1999) e, além de poderem causar anemia em seus hospedeiros (FRYE, 1991; MADER, 1996), podem transmitir hemoparasitos tais como Haemogregarina, Babesiosoma e Trypanosoma (SAWYER, 1986; BARTA et al.,1989; SIDDALL et al., 2001). 
Estudos de transmissão, infecção e parasitemia de hemoparasitos em quelônios através de sanguessugas realizados por Siddall et al. (1990), Molla et al. (2013) e Rossow et al. (2013) evidenciam a capacidade de tais protozoários parasitarem diversas espécies de quelônios. Infecções ocasionadas pelo aparelho bucal de sanguessugas podem ser porta de entrada para hemoparasitos. Sanguessugas que possuem probóscide pertecem a ordem Rhynchobdellida, dividido entre as famílias Glossiphoniidae, Ozobranchidae e Piscicolida (OCEGUERA-FIGUEROA et al., 2014).

Dentre as espécies de hirudíneos, o gênero Placobdella (ERNST, 1971; GRAHAM et al., 1997) representa 50\% de todas as espécies de sanguessugas norte-americanas associadas a quelônios, na América do Sul, encontra-se principalmente os gêneros Unoculubranchiobdella, Desmobdella, Haementeria, Helobdella, Oligobdella e Placobdella em rios brasileiros (CAMPOS-BRITES, 2004; PERALTA et al., 1998; CHRISTOFFERSEN, 2009).

Em Podocnemis expansa (SCHWEIGGER, 1812), há ocorrência da sanguessuga Unoculubranchiobdella expansa, família Ozobranchidae, medindo 3,51 $\mathrm{mm}$ de comprimento e 0,28 $\mathrm{mm}$ de largura com seu corpo distendido (PERALTA et al., 1998). Na espécie P. expansa já foram descritos parasitos do gênero Haemogregarina e Sauroplasma (PICELLI et al., 2015). Nessa circunstância, aos escassos estudos parasitológicos em $P$. expansa, houve a necessidade de verificar se a transmissão de Sauroplasma sp nas populações naturais era ocasionado por sanguessugas devido o vetor ainda ser desconhecido na literatura e o protozoário pouco conhecido. Esta pesquisa teve como objetivo realizar a infecção experimental de Sauroplasma para o quelônio $P$. expansa, mediante repasto sanguíneo de sanguessugas Unoculubranchiobdella expansa.

\section{METODOLOGIA}

O estudo foi conduzido no período de outubro de 2014 a novembro de 2015 . Os exemplares

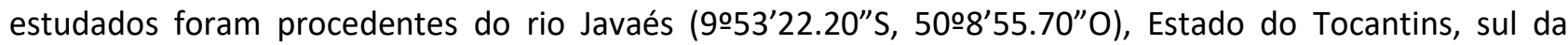
Amazônia brasileira. Esse rio é um dos principais tributários da bacia Araguaia/Tocantins e localiza-se a oeste do Estado, entorno do Parque Nacional do Araguaia, ao norte da llha do Bananal. A base para apoio logístico em campo foi o Centro de Pesquisa Canguçu/CPC da Universidade Federal do Tocantins/UFT, que se situa na região de estudo.

O ecossistema no local é caracterizado pela formação de ecótonos, com a composição de fragmentos típicos de floresta amazônica, pantanal e de um ambiente de cerrado (SOUSA et al., 2012). O clima, segundo a classificação de Köppen-Geiger, é tropical úmido e seco com duas estações bem definidas, o verão, de novembro a abril, meses em que predominam as chuvas, e o inverno, de maio a outubro, período que marca a seca. A precipitação anual fica em torno de $1.800 \mathrm{~mm}$ com temperaturas médias anuais entre 27 e 28 으 (PEEL et al., 2007; SOUSA et al., 2012). 


\section{Captura, marcação e coleta de sangue de $P$. expansa e Sanguessugas}

Durante a pesquisa foram capturados 37 exemplares de $P$. expansa, sendo 17 adultos e 20 filhotes recém eclodidos. Para a captura dos animais adultos empregou-se o arrasto de rede (PORTELINHA et al., 2013) e os filhotes foram coletados através da abertura manual dos ninhos durante o período de eclosão, que no local ocorre de novembro a dezembro (MALVASIO et al., 2002).

Após a captura, os quelônios adultos foram marcados através da perfuração dos escudos marginais da carapaça (CAGLE, 1939; FERNER, 1979). Nos animais capturados, foi realizada a identificação sexual através do entalhe no escudo anal do plastrão (formato de $V$ nas fêmeas e $U$ nos machos) e o tamanho da base da cauda que é relativamente maior nos machos (ERNEST et al., 1989). Os filhotes foram acondicionados em caixas plásticas e levados para o Laboratório de Ecologia e Zoologia (LABECZ) da UFT, onde foram mantidos para a realização das infecções experimentais. A largura da carapaça, o peso, dentre outras variáveis foram medidos nos animais capturados segundo a metodologia de Malvasio et al. (2002).

Para verificar a presença de ectoparasitos nos quelônios adultos realizou-se o exame visual da pele da cabeça, membros e cauda de cada animal (figura 1). As sanguessugas encontradas foram retiradas dos animais com o auxílio de uma espátula e identificadas através de caracteres morfológicos (PERALTA et al., 1998) em estereomicroscópio (40x). Posteriormente, foram acondicionadas em tubos Falcon de $50 \mathrm{ml}$, contendo10 $\mathrm{ml}$ de água do rio Javaés, e levadas vivas para o LABECZ (SAWYER, 1986). A intensidade de sanguessugas (o número de sanguessugas encontrados por hospedeiro infectado) foi usado para descrever a infestação de sanguessugas por tartaruga (RÓZSA et al., 2000).

Os animais adultos e filhotes foram submetidos à coleta de amostras de sangue a partir da veia da cauda ou ventral ou o seio subcarapacial e colocadas em tubos revestidos com heparina de lítio (HERNANDEZDIVERS et al., 2002) para esfregaços sanguíneos. Os mesmos foram fixados com metanol e corados com Giemsa a $10 \%$ (EISEN et al., 2000).

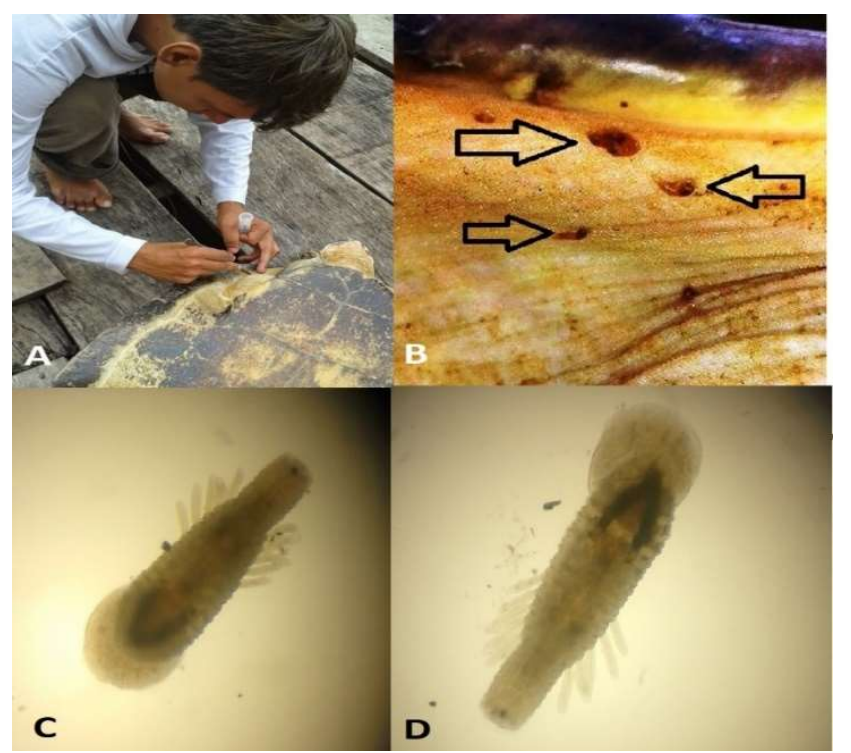

Figura 1: Coleta de Sanguessugas (A). Sanguessugas parasitando uma Tartaruga (B), Sanguessuga Unoculubranchiobdella expansa (C, D). 
Os dados morfométricos das formas parasitárias encontradas foram registrados e expressos em $\mu \mathrm{m}$. O nível de parasitemia foi estimada pela contagem das formas parasitárias em 20 réplicas de 100 eritrócitos (RBC) por campo, totalizando aproximadamente 2.000 eritrócitos (GODFREY et al., 1987). Uma fêmea de $P$. expansa com elevada parasitemia (36 parasitas/2000 eritrócitos) foi selecionada e levada para o cativeiro experimental do LABECZ (SIDDALL et al., 1991), os demais quelônios adultos foram soltos no local de coleta.

\section{Transmissão experimental}

Para a realização das infecções experimentais, a fêmea adulta de $P$. expansa foi isolada em um tanque de fibra de 1,52 m de diâmetro e 0,72 m de profundidade, com capacidade para 1.000 litros de água. Enquanto os 20 filhotes foram colocados separadamente em recipientes plásticos com $50 \mathrm{~cm}$ de diâmetro e $27 \mathrm{~cm}$ de profundidade, com 10 litros de água (figura 2). Contudo, devido ao estresse do transporte e do confinamento 11 filhotes morreram durante as primeiras semanas do estudo. Ademais, ao final desta pesquisa os filhotes foram agrupados em um único tanque com as mesmas dimensões do tanque onde se encontrava a fêmea adulta. Durante a manutenção dos animais em laboratório, a água era trocada regularmente a cada 48 horas e alimentados em igual período com ração de peixe contendo proteína a 30\%.

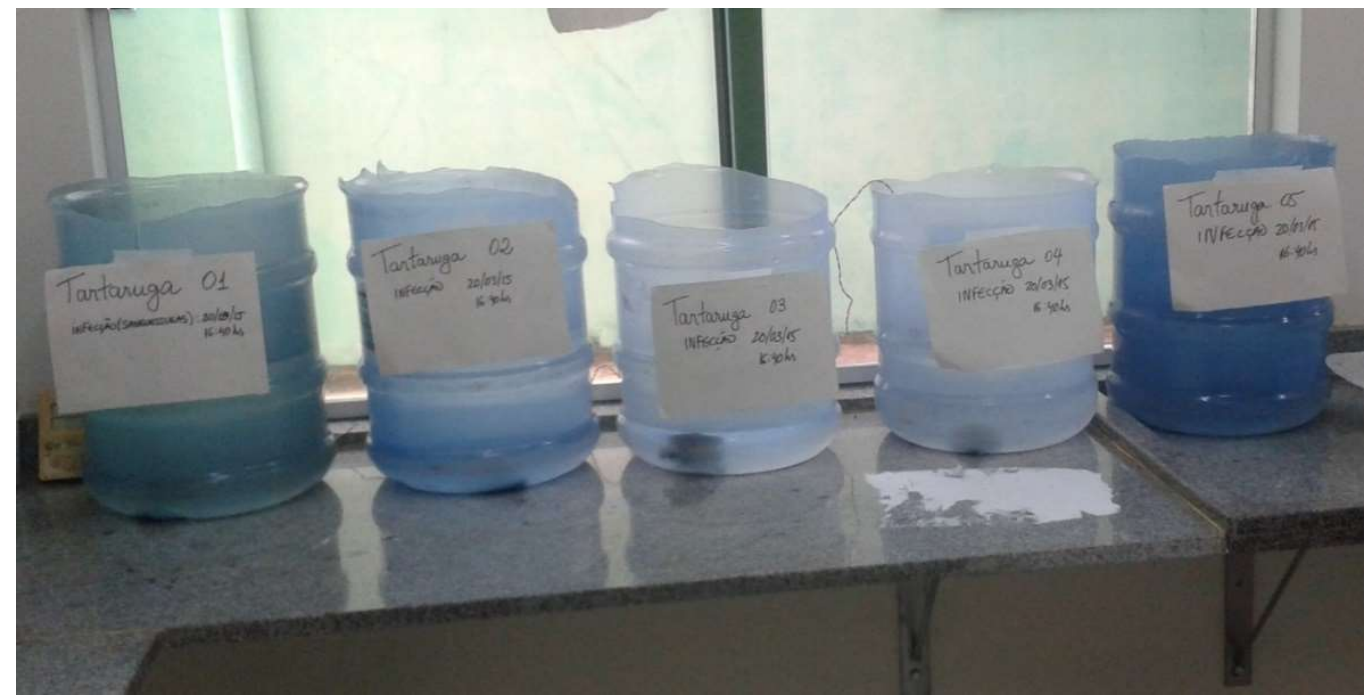

Figura 2: Tanques dos filhotes.

Das sanguessugas levadas ao laboratório, 100 foram colocadas com o auxílio de uma espátula sobre a pele da fêmea adulta de $P$. expansa para a realização do repasto sanguíneo e também para a manutenção dos hirudíneos vivos durante o estudo. Após 10 dias, 54 sanguessugas foram retiradas do espécime adulto e colocadas sobre a pele dos nove filhotes (figura 3), que estavam negativos para Sauroplasma sp. Cada filhote foi exposto ao repasto sanguíneo por 2 sanguessugas durante 5 dias (SIDDALL et al., 2001). Esse procedimento foi realizado por três vezes entre o período de 10 a 20 de abril de 2015 . Foram realizadas amostragens sanguíneas periódicas, a cada 15 dias, como descrito no início da metodologia nos filhotes, para procura de formas parasitárias sanguíneas (PAPERNA, 1989; SIDDALL et al., 2001). 


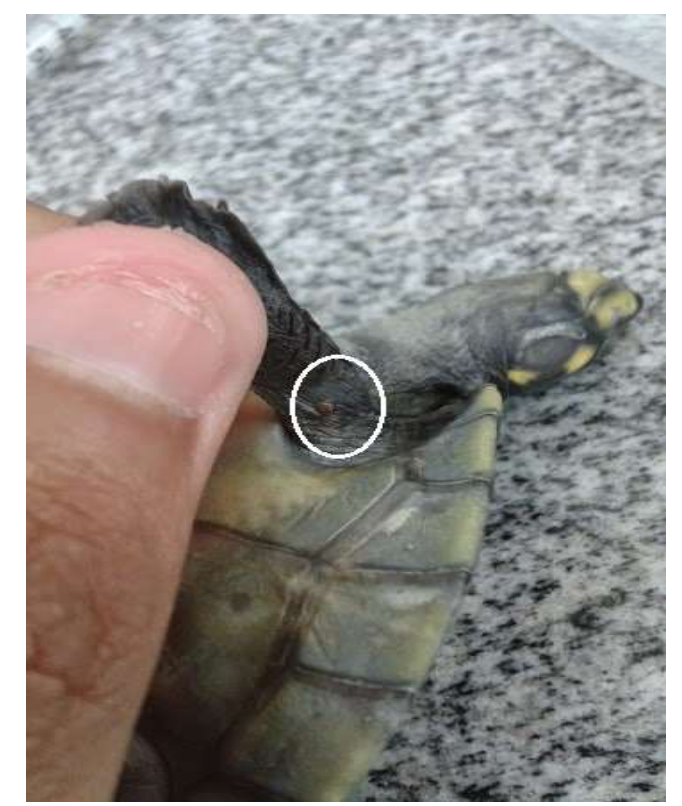

Figura 3: Sanguessuga em filhote de $P$. expansa.

Esta pesquisa contou com as autorizações do Instituto Brasileiro do Meio Ambiente e dos Recursos Naturais Renováveis - IBAMA (SISBIO № 46928-1/ 2014) e do Comitê de Ética em Experimentação Animal da Universidade Federal de Tocantins (№ 23101.002459/2015-99).

\section{Análises estatísticas}

Os dados não apresentam uma distribuição normal por testes D' Agostino foram analisadas por testes não paramétricos (ZAR, 1996). Para determinar possíveis correlações entre a intensidade de sanguessuga e o índice de condição corporal dos quelônios (peso corporal em quilogramas dividido pela largura da carapaça em $\mathrm{cm}$ ) foi utilizado o coeficiente de correlação de Spearman (rs). A regressão logística simples foi usada para determinar se havia uma relação entre a prevalência de Sauroplasma sp. e intensidade de sanguessuga. 0 software BioEstat 5.0 (AYRES et al., 2007) foi utilizado para a análise estatística. Os valores foram considerados estatisticamente significativos para $p<0,05$.

\section{RESULTADOS}

\section{Prevalência}

Foram visualizadas 454 sanguessugas da espécie Unoculubranchiobdella expansa, em 17 animais capturados no rio Javaés no período de fevereiro a março de 2015. Os indivíduos capturados apresentaram prevalência de $100 \%$ quanto ao parasitismo por sanguessugas e $64,7 \%$ da população estudada estava parasitada por Sauroplasma sp. As formas parasitárias encontradas no estudo foi pequenas inclusões sem a presença de cromatina, na forma de um anel e grande vacúolo no centro. Foram encontradas gametócitos de Haemogragarina sp em todos os animais adultos amostrados.

A intensidade de sanguessugas (figura 4) foi significativamente correlacionada com o índice de condição corporal $\left(n=17 ; r_{s}=0.812 ; p \leq 0.001\right)$. Não houve correlação significativa entre a prevalência de hemoparasitos e a intensidade sanguessuga $\left(\chi^{2}=2.1604\right.$; $\left.d f=1 ; p=0.1416\right)$. 


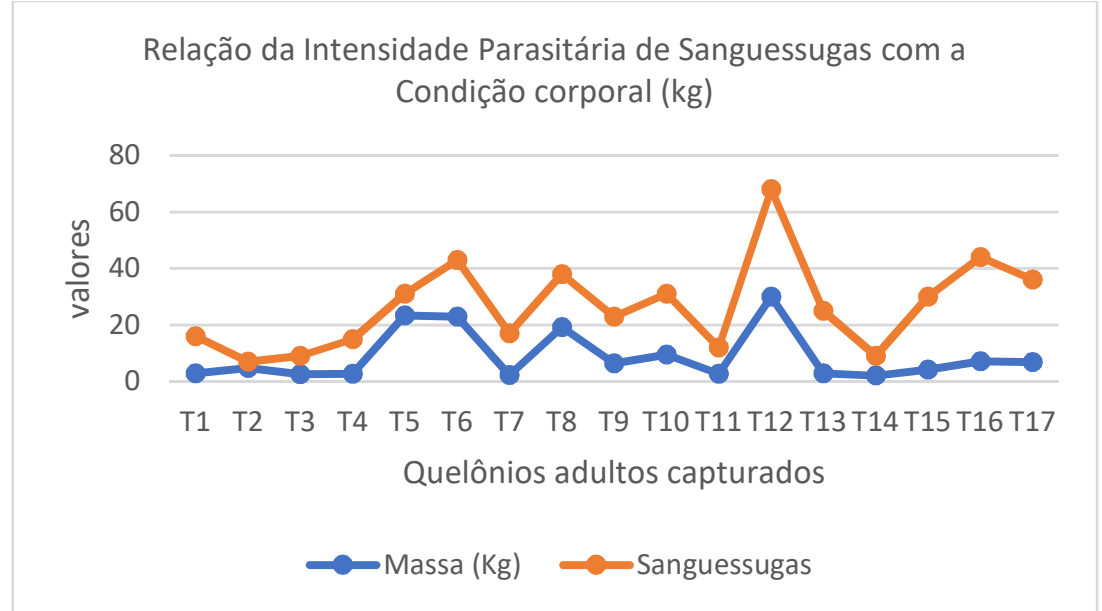

Figura 4: Relação massa corporal com a presença de sanguessugas em indivíduos adultos da amostra pesquisada.

\section{Transmissão experimental}

No mês de maio de 2015 obteve-se resultado positivo para os filhotes submetidos à infecção, apresentando $100 \%$ dos animais infectados por Sauroplasma sp (figura 6), não houve infecção de Hemogregarina $s p$. Observou-se que os índices de prevalência continuaram aumentando nos meses seguintes após o experimento (figura 5).

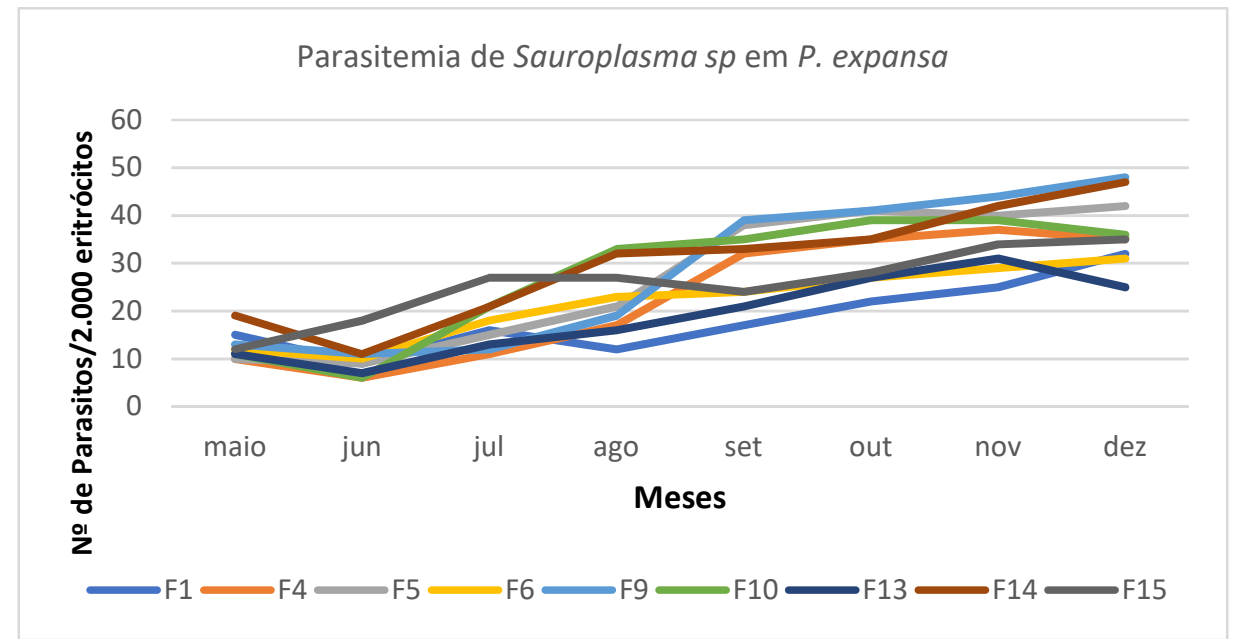

Figura 5: Parasitemia de Sauroplasma sp. em filhotes de Podocnemis expansa no período de maio a dezembro de 2015.

\section{Parasitemia}

Na amostra examinada, constatou-se parasitemia média de 15/2000 (0,75\%) eritrócitos em indivíduos adultos. A parasitemia média dos filhotes foi de 24/2.000 (1,2\%) eritrócitos.

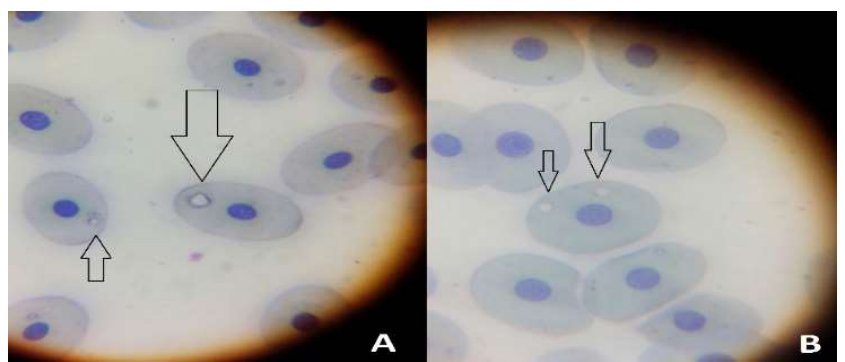

Figura 6: Sauroplasma sp em filhotes de $P$. expansa (A e B). 
Transmissão de Sauroplasma sp. (Piroplasmorida: Haemohormidiidae) pela sanguessuga Unoculubranchiobdella expansa (Hirudinea:

\section{DISCUSSÃO}

\section{Prevalência}

Durante os meses de fevereiro a março, foram encontradas no total 454 sanguessugas nos quelônios capturados. Durante esse período, o rio começa a subir o nível devido à época chuvosa na região, a água permanece mais escura e com maior quantidade de sedimento em sua composição, favorecendo um ambiente mais propício para as sanguessugas, abundantes em locais mais quentes, com rios mais calmos (SILVA et al., 2012).

Observou-se que $P$. expansa habita naturalmente águas mais profundas, animais maiores permanecem mais ao fundo do rio, ocasionado maior parasitismo por sanguessugas, embora os indivíduos com aproximadamente $40 \mathrm{~cm}$ possam ser capturados em locais mais rasos (RYAM et al., 2005; ATAÍDES, 2009; FACHÍN-TERÁN et al., 2004). Segundo McCoy et al. (2007) e Vogt (2008), o tamanho poderia ser um fator determinante na colonização de sanguessugas no hospedeiro, principalmente aquelas espécies que apresentam dimorfismo sexual, variando o tamanho entre machos e fêmeas em algumas espécies de quelônios de água doce. De acordo com Koffler et al., (1978) e Armond (2008), a presença de hirudíneos no ambiente deve estar associada ao período climático da região, temperatura, menor correnteza, dentre outros fatores, relatando diminuição do ectoparasitismo por sanguessugas durante os meses de verão.

A população amostrada dos indivíduos adultos apresentou significativa relação quanto a massa corporal e a presença de sanguessugas, isso se deve a preferência de habitat dos quelônios, jovens habitam geralmente ambientes mais rasos e indivíduos maiores os ambientes categorizados como 'reentrâncias', locais mais profundos dos rios (PORTELINHA, 2010: SALERA JUNIOR et al., 2009) coincidindo com o ambiente das sanguessugas (SILVA et al., 2012).

No referente estudo, os indivíduos adultos apresentaram prevalência de sauroplasma de 64,7\%, relativamente alta. A prevalência de Sauroplasma $s p$ descrita em diversos animais apresenta-se relativamente baixa, estudos em lagartos da Alemanha descrito por Halla et al., (2014) nas espécies Furcifer pardalis, atricollis acanthocercus e Xenagama batillifera apresentaram 7 de 25 (28\%), 5 de 22 (23\%), 5 de 25 (20\%) infectados respectivamente. Segundo Keesing et al., (2006) e Mann (1962), a prevalência de uma infecção emergente está relacionada à riqueza, abundância de hospedeiros além do impacto na população de vetores e parasitos. Segundo Telford (2016; 1984), Siddall et al. (2004), o Sauroplasma (figura 7) possui pouca afinidade com Babesia e Theileria, os piroplasmas encontrados em mamíferos, o que reduz o parasitismo em alguns grupos animais. De acordo com Van (2012), Johnston (1975) e Moller et al., (1990), infecções por Sauroplasma parecem ter pouco efeito visível no próprio hospedeiro.

Estudos voltados para essa finalidade sugerem que aumentando a diversidade de espécies reduz-se o risco de uma doença regulando a abundância de espécies de hospedeiros (seja por competição seja mesmo predação) ou redistribuindo as probabilidades de alimentação dos vetores no caso de doença por eles veiculadas, minimizando a probabilidade das taxas de encontro com o hospedeiro em potencial (HENRIQUES, 2000; BRYGOO, 1966; PEIRCE et al., 1974). 


\section{Transmissão}

A taxa de infecção foi de $100 \%$ nos indivíduos amostrados. Alguns fatores contribuíram para tal resultado, a presença dos hospedeiros disponíveis em um ambiente controlado, a inexistência de competição de ectoparasitas e quantidade de dias de exposição do parasita no hospedeiro. Em condições naturais, a taxa de parasitemia dos parasitos é influenciada pelas diferenças sazonais e ambientais, influenciando na densidade e distribuição dos vetores e hospedeiros (BENNETT et al., 1982; BENNETT et al., 1996).

Segundo Amo et al., (2005) e Carini (1937) a defesa contra parasitas requer uma maior relocação de recursos imunológicos, apenas aqueles com boa condição corporal poderão sobreviver a uma infecção severa, caso contrário poderá reduzir a população embora não há correlações negativas em carga parasitária e condição corporal. Este é o primeiro estudo de infecção experimental de Sauroplasma sp em quelônios de água doce, podendo-se salientar alguns estudos voltados para apenas a ocorrência do parasito em seus hospedeiros.

\section{Parasitemia}

A parasitemia média dos adultos foi de $15 / 2000$ (0,75\%) e dos filhotes foi de $24 / 2.000(1,2 \%)$ dos eritrócitos. Picelli et al., (2016), em seu estudo de Sauroplasma sp em P. expansa, observou diferença significativa na parasitemia entre as três áreas amostradas, cativeiro da Terra Indígena Xambioá (3\%), Fazenda Praia Alta (1.7\%) e dos animais silvestres do rio Javaés (1.8\%), apesar dos resultados, não houve correlação entre a parasitemia, índices eritrocitários e o sexo dos quelônios, reforçando a ideia de que o Sauroplasma sp não seja patogênico (TOIT, 1937; PIENNAR, 1962; SVAHN, 1976), também não foi visto sanguessugas em nenhum dos pontos amostrados.

Rossow et al., (2013) estudando três espécies de quelônios aquáticos na Costa Rica Rinoclemmys funerea, Kinosternon leucostumum e K. scorpioides, não encontraram relação significativa entre parasitemia e condição corporal. Segundo Vanzolini (2003) e Barnard et al., 1994, o parasitismo por ectoparasitos, bem como sua prevalência, sofre influência comportamental das diferentes espécies de hospedeiros uma vez que essas espécies ocupam nichos e ambientes diversificados.

Diferentes espécies apresentam diferenças na suscetibilidade a infecção, essa variabilidade está ligada a fatores genéticos (imunidade a parasitos), comportamentais (comportamento social, agrupamento de colônias), ambientais (pluviometria da região, temperatura) ou à exposição dos parasitos (escolha de habitats, forrageamento, comportamento reprodutivo) (LEE et al., 2006; MARINI et al., 2001; BEGON et al., 2007; MEFFE, 1999; DEEM et al., 2001).

\section{CONCLUSÕES}

O parasitismo por sanguessugas encontradas nas populações de $P$. expansa sofre influência das variações ambientais da região tendo maior infestação no período de cheia do rio. Acredita-se que os vetores 
de Sauroplasma sp sejam as sanguessugas uma vez que os indivíduos submetidos à infecção experimental eram filhotes e não estavam parasitados antes do experimento.

Entender a ecologia parasitária de tais protozoários torna-se um desafio, tendo em vista a gama de hospedeiros que esses hematozoários possuem. Estudos voltados para histologia, análise molecular e genética do vetor pode ser um subsídio para responder as questões voltadas para o parasitismo nas populações naturais.

\section{REFERÊNCIAS}

ALBERTS, A. C.; OLIVA, M. L.; WORLEY, M. B.; TELFORD, S. R. The need for pre-release health screening in animal translocations: a case study of the Cuban iguana (Cyclura nubila). Animal Conservation, v.1, n.3, p.165-172, 1998.

ARMOND, F. N.. Aspectos alimentares e do parasitismo em Podocnemis expansa de ambiente natural e criatório comercial no Estado do Tocantins. Dissertação (Mestrado em Ciências do Ambiente) - Universidade Federal do Tocantins, Palmas, 2008.

AMO, L.; LOPEZ, P.; MARTÍN, J.. Prevalence and Intensity of haemogregarine blood parasites and their mite vectors in the common wall lizard, Podarcis muralis. Parasitology Research. Madrid: Springer-Verlag, v.96, p.378-381, 2005.

ATAÍDES, A. G.. Parâmetros populacionais, aspectos reprodutivos e importância socioeconômica de Podocnemis unifilis, no entorno do Parque Nacional do Araguaia/TO. Dissertação (Mestrado) - Universidade Federal do Tocantins, Palmas, Brasil, 2009.

AYRES, M.; AYRES JUNIOR, M.; AYRES, D. L.; SANTOS, A. A.. Bioestat: Aplicações estatísticas nas áreas das ciências biomédicas. Versão 5.0. Belém: Sociedade Civil Mamirauá, 2007.

BALL, G. H.. Unusual vertebrate and invertebrate hosts of reptilian haemogregarines. In: BALL, G. H.. International Congress Protozoolology. 4 ed. Clemont-Ferrand: Progress in Protozoology, 1973. p.27.

BARNARD, S. M.. A veterinary guide to the parasites of reptiles. Protozoa: Krieger Publishing Company, 1994

BARTA, J. R.; DESSER, S. S.. Development of Babesiosoma stableri (Dactylosomatidae; Adeleida; Apicomplexa) in its leech vector (Batracobdella picta) and the relationship of the dactylosomatids to the piroplasms of higher vertebrates. The Journal of Protozoology, v.36, n.3, p.241-253, 1989.

BEGON, M.; TOWNSEND, C. R.; HARPER, J. L.. Ecologia: de indivíduos a ecossistemas. 4 ed. Porto Alegre: Artmed, 2007.

BENNETT, G. F.; BORRERO H. J. I.. Blood parasites of some birds from Colombia. Journal of Wildlife Diseases, v.12, n.3, p.454-458, 1976.

BENNETT, G. F.; WHITEWAY, M.; WOODWORTH-LYNAS, C. Host-parasite catalogue of the avian haematozoa. Occasional Papers in Biology. Memorial University of Newfoundland, v.5, p.1-243, 1982
BENNETT, R. A.; MADER, D. R.. Cloacal prolapse: Reptile medicine and surgery. Philadelphia: WB Saunders Company, 1996.

BRYGOO, E. R.. Hematozoaires de reptiles malgaches. Notes sur les hémoparasites des Gerrhosauridés de Madagascar avec description d'un trypanosoma nouveau: Trypanosoma betschi parasite de Zonosaurus. Archives de I'Institut Pasteur Madagascar, v.35, p.165-170, 1966.

CAMPOS-BRITES, V. L.; RANTIN, F. T.. The influence of agricultural and urban contamination on leech infestation of freshwater turtels, Phrynops geoffroanus, taken from two areas of the Uberabinha River. Environmental Monitoring and Assessment, v.96, n.1, p.273-281, 2004.

CAGLE, F. R.. A system of marking turtles for future identification. Copeia, v.1939, n.3, p.170-173, 1939.

CARINI, A.. Sur une nouvelle Tunetella d'une tortue du Brésil. Ann. Parasitol. Hum. Comp, v.15, n.6, p.537-538, 1937.

CHRISTOFFERSEN, M. L.. A catalogue of Helobdella (Annelida, Clitellata, Hirudinea, Glossiphoniidae), with a summary of leech diversity, from South America. Neotropical Biology and Conservation, v.4, n.2, p.89-98, 2009 .

DAVIES, A. J.; JOHNSTON, M. R. L.. The biology of some intraerythrocytic parasites of fishes, amphibia and reptiles. Advances in parasitology, v.45, n.1, p.1-107, 2000.

TOIT, P. J.. A new piroplasm (Sauroplasma thomasi ng, n. sp.) of a lizard (Zonurus giganteus, Smith). Onderstepoort J Vet Sci Anim Ind, v.9, p.289-299, 1937.

EISEN, R. J.; SCHALL, J. J.. Life history of a malaria parasite (Plasmodium mexicanum): independent traits and basis for variation. Proceedings of the Royal Society of London B: Biological Sciences, v.267, n.1445, p.793-799, 2000.

ERNST, C. H.. Seasonal incidence of leech infestation on the painted turtle, Chrysemys pitta. Journal of Parasitology, v.57, n.1, p.52, 1971.

FACHÍN-TERÁN, A.; VOGT, R. C.. Estrutura populacional, tamanho e razão sexual de Podocnemis unifilis (Testudines, Podocnemididae) no rio Guaporé/RO, norte do Brasil. Phyllomedusa, v.3, n.1, p.29-42, 2004. 
FERNER, J. W.. Review of marking techniques for amphibians and reptiles. 9 ed. Herpetol Circ, 1979.

FRANK, W.. Sauroplasma and other so-called parasites of reptile blood corpuscles. In: CONGR. PARASITOL. Anais. 1974.

FRYE, F. L.. Applied clinical nonhemic parasitology of reptiles: Biomedical and Surgical Aspects of Captive Reptile Husbandry. Tallahassee: Krieger, 1991.

GODFREY JUNIOR, R. D.; FEDYNICH, A. M.; PENCE, D. B.. Quantification of hematozoa in blood smears. Journal of Wildlife Diseases, v.23, n.4, p.558-565, 1987.

GRAHAM, T. E.; SAUMURE, R. A.; ERICSON, B.. Map turtle winter leech loads. The Journal of parasitology, v.83, n.6, p.1185-1186, 1997.

HALLA, U.; KORBEL, R.; MUTSCHMANN, F.; RINDER, M.. Blood parasites in reptiles imported to Germany.

Parasitology research, v.113, n.2, p.4587-4599, 2014.

HENRIQUES, A. L.. Betrequia ocellata Oldroyd (Diptera, Tabanidae, Rhinomyzini) blood feeding on Caiman crocodilus (Linnaeus)(Crocodylia, Alligatoridae) in Manaus, Brazil. Revista Brasileira de Zoologia, v.17, n.3, p.609-613, 2000.

HERNANDEZ-DIVERS, S. M.; HERNANDEZ-DIVERS, S. J.; WYNEKEN, J.. Angiographic, anatomic and clinical technique descriptions of a subcarapacial venipuncture site for chelonians. Journal of Herpetological Medicine and Surgery, v.12, n.2, p.32-37, 2002.

JOHNSTON, M. R. L.. Distribution of Pirhemocyton Chatton \& Blanc and other, possibly related, infections of poikilotherms. The Journal of Protozoology, v.22, n.4, p.529535, 1975.

KEESING, F.; HOLT, R. D.; OSTFELD, R. S.. Effects of species diversity on disease risk. Ecology letters, v.9, n.4, p.485-498, 2006.

KOFFLER, B. R.; SEIGEL, R. A.; MENDONÇA, M. T. The seasonal occurrence of leeches on the wood turtle, Clemmys insculpta (Reptilia, Testudines, Emydidae). Journal of Herpetology, v.12, n.4, p.571-572, 1978.

LAINSON, R.; LANDAU, I.; SHAW, J. J.. On a new family of non-pigmented parasites in the blood of reptiles: Garniidae fam. nov.,(Coccidiida: Haemosporidiidea). Some species of the new genus Garnia. International Journal for Parasitology, v.1, n.3, p.241-250, 1971.

LEVINE, N. D. CORLISS, J. O.; COX, F. E. G.; DEROUX, G.; GRAIN, J.; LEEDALE, G. F.; LOEBLICH, A. R.; LOM, J.; LYNN, D.; MERINFELD, E. G.; PAGE, F. C.; POLJANSKY, G.; SPRAGUE, V.; VAVRA, J.; WALLACE, F. G.. A newly revised classification of the protozoa. The Journal of protozoology, v.27, n.1, p.37$58,1980$.

LEVINE, N. D.. Some Corrections in Haemogregarine (Apicomplexa: Protozoa) Nomeclature. Journal Protozoology, v.29, n.4, p.601-603, 1982.
LEE, K. A.; MARTIN, L. B.; HASSELQUIST, D.; RICKLEFS, R. E.; WIKELSKI, M.. Contrasting adaptive immune defenses and blood parasite prevalence in closely related Passer sparrows. Oecologia, v.150, n.3, p.383-392, 2006.

LEGLER, J. M.; VOGT, R. C.. Natural Predators na Parasites in: The turtlesos Mexico, Land and fresshwater forms. Sacramento: University of California Press, 2013.

LIGHT, J. E.; SIDDALL, M. E.. Phylogeny of the leech family Glossiphoniidae based on mitochondrial gene sequences and morphological data. The Journal of Parasitology, v.85, n.5, p. 815-823, 1999.

MALVASIO, A.; SOUZA, A. M.; FERREIRA JUNIOR, P. D.; REIS, E. S.; SAMPAIO, F. A. A.. Temperatura de incubação dos ovos e granulometria dos sedimentos das covas relacionadas a determinação sexual em Podocnemis expansa (Schweigger, 1812) e P. unifilis (Troschel, 1848) (Testudines,

Pelomedusidae). Publicações Avulsas, Instituto Pau Brasil de História Natural, n.5, p.11-25, 2002.

MANN, K. H.. Their Structure, Physiology, Ecology and Embryology: International Series of Monographs on Pure and Applied Biology Pergamon Press. Oxford, 1962.

MARINI, M. Â.; DURÃES, R.. Annual cycles of molt and reproduction of passerines from central-south Brazil. Condor, v.103, n.4, p.767-775, 2001.

MCCOY, J.. C.; PRICE, S. J.; FAILEY, E. L.; DORCAS, M. E.. An assessment of leech parasitism on semi-aquatic turtles in the western Piedmont of North Carolina. Southeastern Naturalist, v.6, n.2, p.191-202, 2007.

MEFFE, G. K.. Conservation medicine. Conservation Biology, v.13, n.5, p.953-954, 1999.

MOLLA, S. H.; BANDYOPADHYAY, P. K.; GÜRELLI, G.. On the occurrence of a Haemogregarinae (Apicomplexa) parasite from freshwater turtles of South 24 Parganas, West Bengal, India. Türkiye Parazitolojii Dergisi, v.37, n.2, p.118, 2013.

MOLLER, A. P.; ALLANDER, K.; DUFVA, R.. Fitness effects of parasites on passerine birds: a review. In: MOLLER, A. P.; ALLANDER, K.; DUFVA, R.. Population biology of passerine birds. Berlim: Springer Berlin Heidelberg, 1990. p.269-280.

MURPHY, J. B.; COLLINS, J. T.. Helminth infections and arthropod infestation of captive turtles. In: MURPHY, J. B.; COLLINS, J. T.. A Review of the Diseases and Treatment of Captive Turtle. Lawrence: MAS Publish, 1983. p.56.

OCEGUERA-FIGUEROA, A.; LEÓN-RÈGAGNON, V. Biodiversidad de sanguijuelas (Annelida: Euhirudinea) en México. Revista mexicana de biodiversidad, v.85, n. extra 0 , p.183-189, 2014.

PAPERNA, I.. Developmental cycle of chelonian haemogregarines in leeches with extra-intestinal multiple sporozoite oocysts and a note on the blood stages in the chelonian hosts. Diseases of aquatic organisms, v.7, p.149$153,1989$.

PEEL, M. C.; FINLAYSON, B. L.; MCMAHON, T. A.. Updated world map of the Köppen-Geiger climate classification. 
Hydrology and earth system sciences discussions, v.4, n.2, p.439-473, 2007.

PERALTA, A. S. L.; MATOS, E. R.; SERRA-FREIRE, N. M. Unoculubranchiobdella expansa gen. nov., sp. n. (Hirudinea: Ozobranchidae) parasito de Podocnemis expansa (Chelonia: Pelomedusidae). Entomologia y Vectores, v.5, n.5, p.161177, 1998.

PESSÔA, S. B.; MARTINS, A. V.. Introdução à Parasitologia: Parasitologia Médica. 11 ed. Rio de Janeiro: Guanabara Koogan, 1982.

PEIRCE, M. A.; CASTLEMAN, A. R. W.. An intraerythrocytic parasite of the Moroccan tortoise. Journal of wildlife diseases, v.10, n.2, p.139-142, 1974.

PICELLI, A. M.; CARVALHO, A. V.; VIANA, L. A.; MALVASIO, A.. Prevalence and parasitemia of Haemogregarina sp. in Podocnemis expansa (Testudines: Podocnemididae) from the Brazilian Amazon. Revista Brasileira de Parasitologia Veterinária, v.24, n.2, p.191-197, 2015.

PICELLI, A. M.; CARVALHO, A. V.; VIANA, L. A.; MALVASIO, A.. Parasitization by Sauroplasma sp. (Apicomplexa: Haemohormidiidae) in Chelonian Podocnemis expansa (Testudines: Podocnemididae) in the Brazilian Amazon. The Journal of parasitology, v.102, n.1, p.161-164, 2016.

PIENNAR, U. V.. A preliminary note on reptilian haemoprotozoa in South Africa. In: PIENNAR, U. V.. Haematology of some South African reptiles. Johannesberg: Witwatersrand Univ. Press, 1962. p.186-213

PORTELINHA, T. C.; MALVASIO, A.; PIÑA, C. I.; BERTOLUCI, J.. Reproductive Allometry of Podocnemis expansa (Testudines: Podocnemididae) in Southern Brazilian Amazon. Journal of Herpetology, v.47, n.2, p.232-236, 2013.

PORTELINHA, T. C. G.. Estrutura populacional e alometria reprodutiva de Podocnemis expansa (Testudines, Podocnemididae) no entorno do Parque Nacional do Araguaia, Tocantins. Tese (Doutorado) - Universidade de São Paulo, São Paulo, 2010.

POUGH, F. H.; ANDREWS, R. M.; CADLE, J. E.; CRUMP, M. L; SAVITZKY, A. H.; WELLS, K. D.. Herpetology. 3 ed. Upper Saddle River: Prentice Hall, 2004.

READEL, A. M.; PHILLIPS, C. A.; WETZEL, M. J.. Leech parasitism in a turtle assemblage: effects of host and environmental characteristics. Copeia, v.2008, n.1, p.227233, 2008.

ROSSOW, J. A.; HERNANDEZ, S. M.; SUMNER, S. M.; ALTMAN, B. R.; CRIDER, C. G.; GAMMAGE, M. B.; SEGAL, K. M.; YABSLEY, M. J.. Haemogregarine infections of three species of aquatic freshwater turtles from two sites in Costa Rica. International Journal for Parasitology: Parasites and Wildlife, v.2, n.1, p.131-135. 2013.

RÓZSA, L.; REICZIGEL, J.; MAJOROS, G.. Quantifying parasites in samples of hosts. Journal of Parasitology, v.86, n.2, p.228232, 2000.
RYAN, T. J.; LAMBERT, A.. Prevalence and colonization of Placobdella on two species o freshwater turtles (Graptemys geographica and Sternotherts odoratus). Journal of Herpetology, v.39, n.2, p.284-287, 2005.

SALERA JUNIOR, G.; MALVASIO, A.; PORTELINHA, T. C. G.. Avaliação de padrão irregular dos escudos do casco em Podocnemis expansa e Podocnemis unifilis (Testudines, Podocnemididae). Acta Amazonica, v.39, n.2, p.431-438, 2009.

SAWYER, R. T.. Leech biology and behaviour. Oxford: Clarendon Press, 1986.

SCHNEIDER, L.; FERRARA, C. R.; VOGT, R. C.; BURGER, J. History of turtle exploitation and management techniques to conserve turtles in the Rio Negro Basin of the Brazilian Amazon. Chelonian Conservation and Biology, v.10, n.1, p.149-157, 2011.

SIDDALL, M. E; GAFFNEY, E. S.. Observations on the leech Placobdella ornata feeding from bony tissues of turtles. Journal of Parasitology, v.90, n.5, p.1186-1188, 2004.

SIDDALL, M. E; DESSER, S. S.. Merogonic development of Haemogregarina balli (Apicomplexa: Adeleina: Haemogregarinidae) in the leech Placobdella ornata (Glossiphoniidae), its transmission to a chelonian intermediate host and phylogenetic implications. The Journal of parasitology, v.77, n.3, p.426-436, 1991.

SIDDALL, M. E.; DESSER, S. S.. Transmission of Haemogregarina balli from painted turtles to snapping turtles through the leech Placobdella ornata. Journal of Parasitology, v.87, n.5, p.1217-1218. 2001.

SIDDALL, M. E.; DESSER, S. S.. Gametogenesis and sporogonic development of Haemogregarina balli (Apicomplexa: Adeleina: Haemogregarinidae) in the leech Placobdella ornata. The Journal of Protozoology, v. 37, n.6, p.511-520, 1990.

SILVA, M. S. G. M.; QUEIROZ, J. F.; LOSEKANN, M. E.; MARIGO, A. L. S.; NASCIMENTO, M.. Utilização de coletores com substrato artificial para o biomonitoramento da qualidade da água na aquicultura. São Pauo: EMBRAPA 2012.

SVAHN, K.. A new piroplasm Sauroplasma boreale sp. $n$. Haemosporidia, Theliriidae) from the sand lizard Lacerta agilis. Norw J Zool, v.24, n.1, p.1-16, 1976.

SOUSA, P. A. B.; BORGES, R. S. T.; DIAS, R. R.. Atlas do Tocantins: Subsídios ao planejamento da gestão territorial. Palmas: SEPLAN, 2012.

TELFORD JUNIOR, S. R.. Haemoparasites of reptiles In: HOFF, G. L.; FRYE, F.L., JACOBSON, E. R.. Diseases of amphibians and reptiles. New York: Plenum Press, 1984. p.408-434. TELFORD JUNIOR, S. R.. Hemoparasites of the Reptilia: Color atlas and text. CRC Press, 2016.

UILENBERG, G.; BLANC, C. P.. Note sur un Hematozoaire d'un Reptile malgache, Uroplatus fimbriatus (Gekkonidae): Sauroplasma sp. (Protozoa incertaesedis). Ann Parasitol Hum, v.41, p.209-212, 1966. 
VANZOLINI, P. E.. On clutch size and hatching success of the South American turtles Podocnemis expansa (Schweigger, 1812) and P. unifilis Troschel, 1848 (Testudines,

Podocnemididae). Anais da Academia Brasileira de Ciências, v.75, n.4, p.415-430, 2003.

VAN, J. A. S.. Ecology, taxonomy and possible life cycles of blood protozoans infecting crag lizards (pseudocordylus spp.) From the eastern free state highlands. Thesis (Ph.D. Zoology and Entomology) - University of the Free State, 2012.

VOGT, R. C.. Tartarugas da Amazônia. 1 ed. Manaus: Instituto Nacional de Pesquisa da Amazônia, 2008.

ZAR, J. H.. Biostatistical Analysis. 3 ed. Upper Saddle River: Prentice-Hall, 1996.

A CBPC - Companhia Brasileira de Produção Científica (CNPJ: 11.221.422/0001-03) detém os direitos materiais desta publicação. Os direitos referem-se à publicação do trabalho em qualquer parte do mundo, incluindo os direitos às renovações, expansões e disseminações da contribuição, bem como outros direitos subsidiários. Todos os trabalhos publicados eletronicamente poderão posteriormente ser publicados em coletâneas impressas sob coordenação da Sustenere Publishing, da Companhia Brasileira de Produção Científica e seus parceiros autorizados. Os (as) autores (as) preservam os direitos autorais, mas não têm permissão para a publicação da contribuição em outro meio, impresso ou digital, em português ou em tradução. 